\title{
Neck Muscle Vibration and Spatial Orientation During Stepping in Place in Humans
}

\author{
MARCO BOVE, ${ }^{1}$ GREGOIRE COURTINE, ${ }^{2}$ AND MARCO SCHIEPPATI ${ }^{3}$ \\ ${ }^{1}$ Department of Experimental Medicine, Section of Human Physiology, University of Genoa, I-16132 Genoa, Italy; \\ ${ }^{2}$ Institut National de la Santé et de la Recherche Médicale, Motricité \& Plasticité, University of Burgundy, F-21078 Dijon, \\ France; and ${ }^{3}$ Physiological Institute, University of Pavia, and Human Movement Laboratory, Fondazione Salvatore \\ Maugeri, Istituto di Ricovero e Cura a Carattere Scientifico, I-27100 Pavia, Italy
}

Received 18 March 2002; accepted in final form 15 July 2002

\begin{abstract}
Bove, Marco, Gregoire Courtine, and Marco Schieppati. Neck muscle vibration and spatial orientation during stepping in place in humans. J Neurophysiol 88: 2232-2241, 2002; 10.1152/jn.00198.2002. Unilateral long-lasting vibration was applied to the sternomastoid muscle to assess the influence of asymmetric neck proprioceptive input on body orientation during stepping-in-place. Blindfolded subjects performed 3 sequences of 3 trials, each lasting $60 \mathrm{~s}$ : control, vibration applied during stepping (VDS), and vibration applied before stepping (VBS). VDS caused clear-cut whole body rotation toward the side opposite to vibration. The body rotated around a vertical axis placed at about arm's length from the body. The rotation did not begin immediately on switching on the vibrator. The delay varied from subject to subject from a few seconds to about $10 \mathrm{~s}$. Once initiated, the angular velocity of rotation was remarkably constant (about $1 \%$ s). In VBS, at the beginning of stepping, subjects rotated for a while as if their neck were still vibrated. At a variable delay, the direction of rotation reversed, and the effects were opposite to those observed during VDS. Under no condition did head rotation, head roll, or lateral body tilt accompany rotation. The results confirm and extend the notion that the neck proprioceptive input plays a major role in body orientation during locomotion. The body rotation does not seem to depend on the same mechanisms that modify the erect posture; rather, the asymmetric neck input would seem to modify the egocentric bodycentered coordinate system.
\end{abstract}

\section{N T R O D U C T I O N}

Muscle tendon vibration is an adequate stimulus for the spindle receptor. The small but fast vibration cycles almost selectively induce a train of action potentials in the primary endings connected to the large-diameter group Ia afferent fibers. The discharge follows one-to-one the vibration cycles within a wide range and lasts as long as vibration continues (Burke et al. 1976; Roll et al. 1989a). Both human primary and secondary spindle endings respond to vibration stimuli (Burke et al. 1976). However, the Ia afferent fibers from the muscle primary spindles are much more sensitive to vibration and can be driven to higher frequency rates than the secondaries, which normally respond at a sub-harmonic of the vibration.

Subjects can perceive an illusion of limb movement in the direction that would lengthen the vibrated muscles: the spindle discharge is interpreted as muscle lengthening and limb dis-

Address for reprint requests: M. Schieppati, Centro Studi Attività Motorie (CSAM), Fondazione Salvatore Maugeri (IRCCS), Via Ferrata 8, I-27100 Pavia, Italy (E-mail: mschieppati@fsm.it and marco.schieppati@unipv.it). placement (Craske 1977; Goodwin et al. 1972; Roll and Vedel 1982). Interestingly, motor rather than somatosensory areas seem to convey the illusion of limb movement (Naito et al. 1999). This input, however, does not necessarily produce explicit perceptions. It can produce an error in motor coordination; for example, vibration of an arm muscle involved in a movement sequence (hand opening during elbow extension) caused subjects to open the hand before the elbow reached a given angle (Cordo et al. 1995). Furthermore, it can affect the generation of an egocentric body-centered coordinate system. Neck muscle vibration elicits apparent motion of a stationary visual target and deviation of the perceived "straight ahead" (Biguer et al. 1988; Karnath et al. 1994, Strupp et al. 1999; Taylor and McCloskey 1991). In "neglect" patients, neck muscle vibration can compensate the horizontal displacement of the sagittal midplane (Karnath 1994; Karnath et al. 1993). In standing subjects, neck muscle vibration induces body tilt and increased sway, suggesting that posture is organized with respect to a "body schema," to the construction of which neck input contributes together with eye and skeletal muscle (Eklund 1972; Gurfinkel et al. 1995; Ivanenko et al. 1999; Kavounoudias et al. 1999; Roll et al. 1989b; Smetanin et al. 1993). Most likely, the parietal cortex contributes to the egocentric representation of space, since many of its areas receive signals from neck muscles and from the labyrinth (Bottini et al. 2001).

The proprioceptive input from the neck is not only integrated in the control of stance, but also in the steering of locomotion. Lund (1980) originally reported that it was possible to modify standing posture, as well as to induce walking, by applying neck muscle vibration. Fukuda (1959) and Ushio et al. (1976) reported that stepping on the spot was affected by static head inclination and neck torsion, respectively. Ivanenko et al. (2000) showed that if the head is horizontally turned or the eyes are laterally rotated, vibration of dorsal neck muscles during stepping-in-place causes stepping in the direction of the naso-occipital axis or of the gaze, respectively. Preliminary findings from this laboratory have shown that lateral neck muscle vibration, applied during short distance walk on firm ground in the absence of vision, produces undershoot of target

\footnotetext{
The costs of publication of this article were defrayed in part by the payment of page charges. The article must therefore be hereby marked "advertisement" in accordance with 18 U.S.C. Section 1734 solely to indicate this fact.
} 
and deviation of gait trajectory toward the site opposite to vibration (Bove et al. 2001).

The aim of this study was to describe the effect of asymmetric neck muscle vibration on the body orientation occurring during an extended stepping task. At variance with ground locomotion, stepping in place has the advantage that prolonged periods of locomotor-like activity can be observed and recorded by means of a movement-analysis system. The questions to be addressed were as follows: 1 ) how does long-term neck vibration affect body orientation; 2) does vibration induce any perception or illusion of body rotation, which might entrain reactive steering of the body; and 3) is rotation preceded or accompanied by head or trunk postural changes? An answer to these questions would contribute to our understanding of the mechanisms responsible for the steering effects of vibration on locomotion and help define some aspects of the way in which proprioceptive input from the neck contributes to the generation of a reference system necessary for allowing the operation of the superimposed mechanisms of visuo-motor coordination during navigation.

\section{METHODS}

\section{Subjects}

Six subjects (4 males and 2 females; age range, 26-35 yr; mean age, $30.4 \mathrm{yr}$ ) volunteered for these experiments. They had no history of neurological diseases or vestibular disorders, no signs or symptoms of cervical diseases, and no discrepancies between right and left lower limbs that can affect veering of locomotion (Boyadjian et al. 1999). Subjects gave written and informed consent, and the study conformed with The Declaration of Helsinki.

\section{Procedure}

Subjects stood blindfolded and barefoot in the middle of the experimental field in a darkened room, with the orientation of feet, trunk, and head aligned. Subjects practiced stepping-in-place, with eyes open and closed, for about $1 \mathrm{~min}$ before the recording session. They were then instructed to start stepping-in-place at their own preferred pace, on a verbal go-signal, until told to stop after $60 \mathrm{~s}$. This was done under both control and vibration conditions, in which case they were told not to react to the applied perturbation. Thereafter, subjects performed three sequences of three trials each, in this order: no vibration (Control), vibration applied during stepping (VDS), and vibration applied before stepping (VBS). Under all conditions, the onset of acquisition of the kinematics signal started about $3 \mathrm{~s}$ after the verbal go-signal for stepping onset. By this time, subjects had already performed one complete stepping cycle. Subjects stepped in place for the entire epoch of acquisition (60 s). For VDS, the vibrator was set concurrently with the onset of the acquisition. The vibrator and the acquisition were shut off $60 \mathrm{~s}$ later at the end of the trial. For VBS, a 1-min vibration was administered to the blindfolded standing subjects, and vibration-off was the signal to start stepping. Three- to 6-min rest interval separated each successive trial, during which subjects were free to move. Between trials and sessions, the blindfold was not removed; one investigator passively moved the subject randomly across the room, to "cancel" any possible new reference they might have constructed. Subjects were then helped to return to the starting position for the successive trial. Three trials were performed for each control or vibration session, each session lasting about $10 \mathrm{~min}$. Ten minutes elapsed between sessions. For both vibration conditions, separate sessions were performed with vibration on the left (VDS 1 and VBS 1) and right side of the neck (VDS $r$ and VBS r) on a different day. Under no circumstances did the subjects report sensations of head turning or tilting in response to the vibration, nor was actual head turning or tilting observed by the experimenters. On a different day, subjects performed stepping-in-place trials, blindfolded with no vibratory stimulus, with the head deliberately kept rotated about $50^{\circ}$ on the right and left side. This test was done to verify the effects of the head rotation on body orientation during stepping. Subjects repeated three trials for each condition (head in primary position, rotation left, and rotation right).

\section{Stimulation}

The vibrator consisted of a DC motor with an eccentric on the shaft embedded in a plastic tube with a diameter of $3 \mathrm{~cm}$ and a length of 6 $\mathrm{cm}$ (Dynatronic, Valence, France). It was set to deliver an $80-\mathrm{Hz}$ vibration, at which frequency it exerted an oscillating force ranging between -2.5 and $2.5 \mathrm{~N}$ in the direction normal to the skin surface overlying the sternomastoid muscle, as measured by tightly binding the vibrator to a strain gauge. The vibrator was applied to the side of the cervical column (left and right in separate sessions) by means of an elastic strap that passed around the neck. It was fixed over the belly of the sternomastoid muscle, about $40 \%$ of its length below its insertion on the mastoid, i.e., about $4 \mathrm{~cm}$ below the mastoid bone along the muscle length and $2 \mathrm{~cm}$ anterior to a frontal plane passing through the mastoid apex (or about $5 \mathrm{~cm}$ lateral and $7 \mathrm{~cm}$ anterior to the cervical spine). The cylinder axis was normal to the direction of muscle and exerted an acceleration normal to the muscle surface between -4.45 and $4.45 \mathrm{~g}$, measured by a three-axial accelerometer (TSD109 BIOPAC Systems, Santa Barbara, CA). The vibrator was put in place at the beginning of the experiment and kept in place throughout the session. To estimate the propagation of the vibration to the temporal bone, which could possibly induce activation of the labyrinthine receptors, we placed two accelerometers with their axes about parallel to the semicircular canals: one on the vibrator located on sternomastoid muscle and the other on the ipsilateral mastoid bone. The amplitude of the main peak in the power spectrum of the largest of the three output signals recorded at the mastoid bone was on average $<1 \%(0.66 \pm 0.03 \%)$ of the same signal measured at the sternomastoid muscle. This finding would speak against a major propagation of the mechanical wave to the labyrinth, and indirectly lessen the likelihood of vestibulum-mediated vibration effects.

\section{Recording}

Body movements were recorded by an optoelectronic motion analysis system (ProReflex; Qualisys AB, Sävedale, Sweden). Four infrared cameras were located around the experimental field, identifying a space of about $2.5 \times 2.5 \times 2.5 \mathrm{~m}$. The reflective markers were attached to the skin on the forehead, vertex of the head, left and right acromions, and on the right hemi-body: anterior superior iliac spine, lateral femur epicondyle, lateral malleolus, and fifth metatarso-phalangeal joint (Fig. 1A). The markers' position was sampled at a frequency of $100 \mathrm{~Hz}$.

\section{Data analysis}

The following parameters were computed for each trial under each condition. 1) Step cycle frequency. The trace of the vertical displacement of the knee versus time was subjected to a fast Fourier analysis. The value of the main peak was taken as the mean value of step frequency. 2) Antero-posterior and medio-lateral final positions. The initial antero-posterior axis of the subjects corresponded to the $x$ axis of the experimental field and the medio-lateral corresponded to the $y$ axis. The first and last 100 samples of the vertex marker at the beginning and the end of each trial were averaged: the mean position of the head during the first and last stepping cycles minimizes the error connected with head oscillation. The averaged initial position was then subtracted from the averaged final position. 3) Path length. 
A

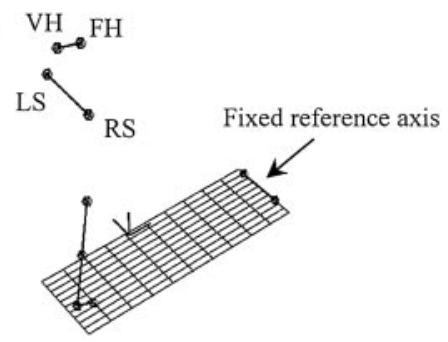

$0 \mathrm{~s}$

B

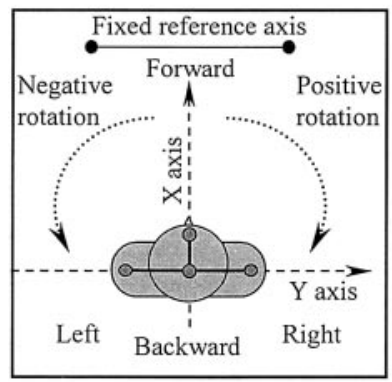

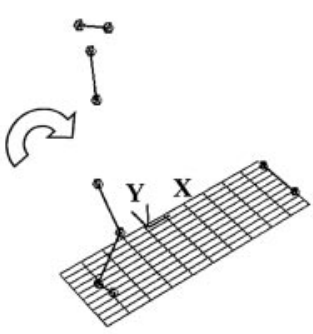

$30 \mathrm{~s}$

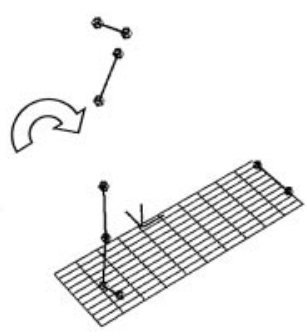

$60 \mathrm{~s}$

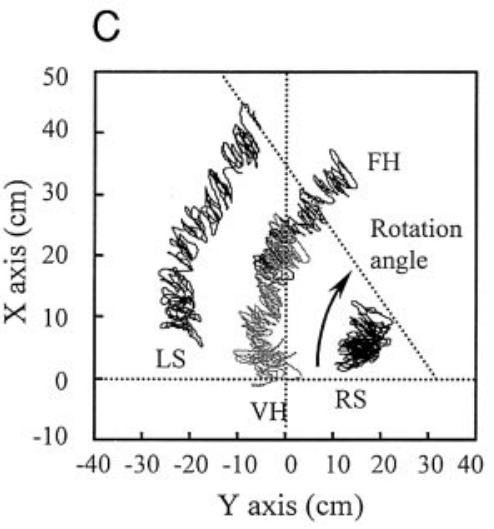

D

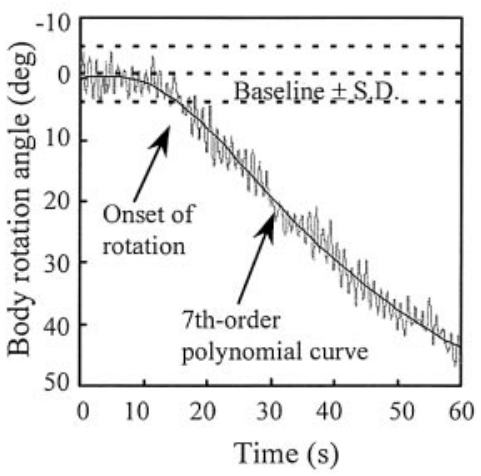

FIG. 1. Experimental methods. A: stick diagram represents the body orientation of a subject against time, when the vibration was delivered on the left side of the neck during stepping-in-place. Closed circles are the reflective markers, recorded by the 4 infrared cameras and positioned on forehead $(\mathrm{FH})$, vertex of the head (VH), left and right shoulders (LS and RS), and on the right anterior superior iliac spine, lateral femur epicondyle, lateral malleolus, and 5th metatarso-phalangeal joint. After an initial delay, the subject rotated clockwise, i.e., toward the direction opposite to the position of vibrator. $B$ : subject was positioned at a starting point around the zero coordinates of the $x-y$ axes with the shoulders axis almost parallel to the fixed reference axis. Forward and backward displacement were defined as positive and negative, respectively. Rotation angles on the right or left side were positive or negative, respectively. $C$ : top-down view of the stepping path described by the markers positioned on the FH and VH (dark and light gray, respectively) and on RS and LS (black). D: time course of the change in the angle defined by the shoulder axis and the fixed reference axis. Baseline \pm SD represents a positive and a negative threshold. Onset of the rotation was indicated with the crossing of these thresholds by the polynomial fitting curve.

This was the path traveled by the marker placed on head vertex during the 60 -s epoch. The cyclic medio-lateral oscillations were eliminated by fitting a 7 th order polynomial curve to the original data set and the length of the curve was computed (Fig. 1D). 4) Body rotation. It was represented by the changes in the angle defined by the shoulder axis and a fixed reference axis (Fig. 1A). This reference was identified by two markers put on the floor at a distance from the stepping subject and parallel to the initial shoulder axis ( $y$ axis; Fig. $1 B$ ). The first and last 100 samples $(1 \mathrm{~s})$ of the angle values at the beginning and the end of each epoch were averaged. The initial position was then subtracted from the averaged final position. The body rotation was around zero in the control condition and could become negative or positive if the neck was vibrated, on the right or left side of the neck, respectively. Negative value indicated a counter-clockwise; positive value was a clockwise rotation (Fig. 1C). 5) Latency of body rotation. This was evaluated off-line for the VDS condition. The function rotation-angle versus time were fitted with a 7 th-order polynomial (Fig. 1D). A positive and a negative threshold were used to indicate the emergence of the rotation effect induced by the vibration (Fig. 1D). For each subject, the thresholds were set at the SDs around the mean body rotation angle, calculated for the six control trials from the entire epochs. 6) Velocity of body rotation. It was evaluated from the onset of rotation in the VDS condition. The function angle of rotation versus time was fitted with a straight line from the rotation onset to the end of the trial, since observation of the data indicated that the angular velocity was approximately constant. This was confirmed by computing the correlation coefficient of the linear fitting. 7) Radius of curvature. The curvature of the path traveled by the head vertex during the vibration-induced rotation was approximated by fitting an arc of circumference to the data points and calculating its radius. The center of the circumference and the radius gave an indication of the virtual point, around which the body turned during rotation. 8) Head-shoulder angle. This was the mean angle between head antero-posterior axis and shoulder medio-lateral axis. For VDS, to sharpen the analysis, the mean angle was computed separately only during the period of rotation, i.e., from the time of onset of rotation identified as above, since the angle in the period preceding the rotation in the VDS condition did not significantly change with respect to the control (one-way ANOVA: $d f=2,51 ; F=0.39 ; P=0.67)$. 9) Medio-lateral and antero-posterior body inclination. The average distance, between the markers on the right shoulder and malleolus, both projected on a horizontal plane, was measured. This was taken as an index of medio-lateral inclinations of the body during stepping-in-place, since the line joining the shoulders did not change its position across conditions (the change in vertical distance between right and left shoulder with respect to control was: VDS $1:-2.08 \pm 1.01 \mathrm{~mm}$, VDS $r:-0.02 \pm 2.15$; one-way ANOVA: $d f 2,69 ; F=1.023 ; P=0.21$ ). Thus increase and decrease of the shoulder-malleolus distance indicated inclination toward the right and left side, respectively. Anteroposterior deviations between the markers on the right shoulder and malleolus were also evaluated to check for possible shoulder inclination forward or backward with respect to the malleolus. The inclination during stance was also recorded (sampling frequency $10 \mathrm{~Hz}$, trial duration $51.2 \mathrm{~s}$ ) by means of dynamometric platform (QFP Systèmes, 
Mougin, France) to compare the changes in center of foot pressure (CFP) with the medio-lateral and antero-posterior body inclination. The lateral and antero-posterior displacement (CFP) was measured 1) without vibration (Control); 2) during vibration of the right (VDS r), and 3 ) left side (VDS 1). The trials were spaced by at least 3 min, to avoid the effects of long-lasting vibration and repetition of trials (Tarantola et al. 1997). 10) Lateral tilt (roll) of head on trunk. Roll was evaluated, for all subjects and for all vibration trials, by computing the distances between the markers placed on the right shoulder and the vertex of the head and between the left shoulder and the vertex of the head. We assumed that head roll would be attested by the following: 1) a difference in the mean lengths of these distances during the whole duration of the epoch exhibiting turning behavior (vibration during stepping) with respect to control condition; 2) a difference of these distances within the same turning epoch, between the first seconds (showing no turning) and the last few seconds (showing a clear cut ongoing rotation).

\section{Statistical analysis}

Before ANOVA, a test on the homogeneity of the variances was done (Levene's test). In the case of a nonhomogeneity, the nonparametric Mann-Whitney $U$ test was used to assess differences among the three conditions. Otherwise, the effects of vibration on stepping frequency, antero-posterior and medio-lateral position of the trajectory endpoint, path length, head-shoulder angle, medio-lateral body inclination, and displacement of CFP were assessed by ANOVA. This was normally made for the means of the populations represented by all trials (all subjects collapsed). The post hoc Newman-Keuls test was employed to assess differences among Control, VDS, and VBS conditions for both stepping-in-place and quiet-stance protocol. All averaged data are presented as mean \pm SE. The level of significance was set at $P<0.05$.

\section{R E S U L T S}

\section{Stepping frequency (VDS and VBS)}

Neck muscle vibration administered both during (VDS) and for $60 \mathrm{~s}$ before stepping (VBS) did not modify the frequency with respect to Control. There was no evidence of systematic changes (one-way ANOVA: $d f=2,105 ; F=0.75 ; P=0.47$ ) when the vibration was administered either during or before stepping (Control 1: $0.86 \pm 0.02 \mathrm{~Hz}$; VDS 1: $0.87 \pm 0.016 \mathrm{~Hz}$; and VBS 1: $0.87 \pm 0.014 \mathrm{~Hz}$. Control r: $0.87 \pm 0.023 \mathrm{~Hz}$; VDS r: $0.85 \pm 0.02 \mathrm{~Hz}$; and VBS r: $0.88 \pm 0.02 \mathrm{~Hz}$ ).

\section{Antero-posterior and medio-lateral final position (VDS and $V B S$ )}

The effect of the vibration on the capacity of the subjects to keep stepping on the spot was evaluated by comparing their initial and the final position. Under Control, VDS, and VBS conditions, the final position of the body was of about 33,31 , and $26 \mathrm{~cm}$ ahead of the initial position, respectively; no significant difference across conditions was present (one-way ANOVA: $d f=2,105 ; F=0.55 ; P=0.58)$. In the mediolateral direction, VDS produced a significant displacement to the side opposite to the vibrator (one-way ANOVA: $d f=5$, $102 ; F=5.96 ; P<0.001$ ). Displacements of similar amplitude but different sign were observed when the vibration was administered to the left and right side (Control 1: $1.89 \pm 1.19$ $\mathrm{cm}$; VDS 1: $19 \pm 6.04 \mathrm{~cm}$; and VBS 1: $-7.05 \pm 3.05 \mathrm{~cm}$. Control r: $-1.5 \pm 1.55 \mathrm{~cm}$; VDS r: $-18.31 \pm 7.15 \mathrm{~cm}$; and VBS r: $7.17 \pm 5.76 \mathrm{~cm}$ ). Under VBS condition, a minor lateral displacement, not significantly different from Control, was observed toward the same side of the vibration (analyzed separately for left and right side).

\section{Path length (VDS and VBS)}

A curvilinear trajectory of the head vertex was the rule; subjects tended to slowly turn on the spot while stepping and never showed any pure translation, the final position being a trade-off between a consistent body rotation and a slight component of translation accompanying the course of the rotation. There was a systematic increase of the path length in the two vibration conditions (Control: $30.3 \pm 4.1 \mathrm{~cm}$; VDS: $62.98 \pm$ $6.14 \mathrm{~cm}$; VBS: $51.52 \pm 4.81 \mathrm{~cm}$; one-way ANOVA: $d f=2$, $105 ; F=12.89 ; P<0.001)$. The post hoc test assessed differences between VDS and Control $(P<0.001)$ and VBS and Control $(P<0.001)$. No difference was observed between the two vibration conditions. Since stepping frequency was unchanged by vibration, we assumed that neck vibration induced an increase in step length. By dividing the velocity of the trajectory by the stepping frequency across all trials, the step length proved to be $0.65 \pm 0.06 \mathrm{~cm}$ in the Control, $1.3 \pm 0.11$ $\mathrm{cm}$ in the VDS, and $1.1 \pm 0.1 \mathrm{~cm}$ in the VBS condition.

\section{Body rotation during vibration (VDS)}

The slow yet continuous body rotation was the main and consistent effect of the lateral neck vibration induced in the stepping subjects. During VDS, such rotation could start, from trial to trial, at variable latency from the vibration onset. Figure $1 A$ shows the time-evolution of the stick diagram of a representative subject, when the vibration was delivered on the left side: the subject rotated clockwise, i.e., opposite to the position of vibrator. Figure 2 summarizes the effects of the vibration on the body orientation under all the experimental conditions, subject per subject, and within all the subjects. Under Control conditions, subjects exhibited only a slight forward displace-

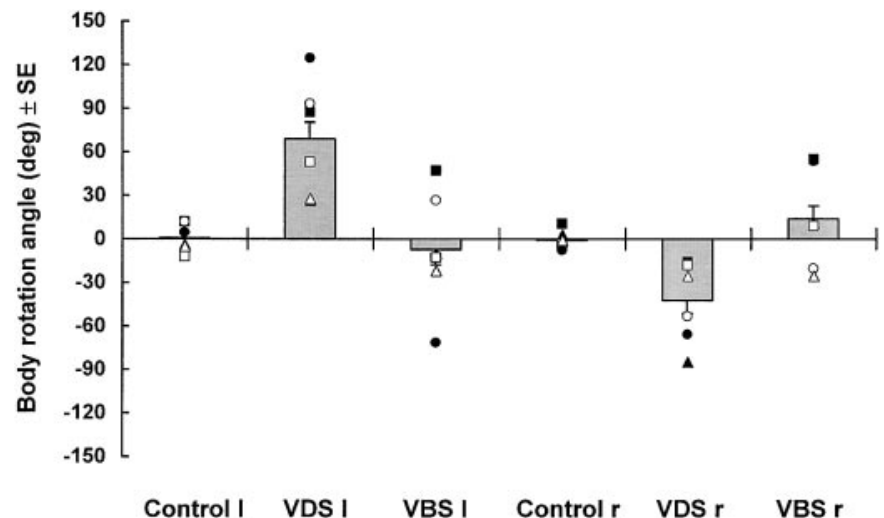

FIG. 2. Effects of vibration on the body rotation under all the experimental conditions. These are showed subject per subject (symbols) and for all the subjects (grand averages $\pm \mathrm{SE}$ ) and separately for vibration side (Control 1 , VDS 1, VBS 1: left side vibration; Control r, VDS r, VBS r: right side vibration). In control condition, subjects did not rotate during stepping. In the VDS condition, vibration made all subjects undergo a systematic and major rotation of the body in the direction opposite to the side of vibration. VBS was characterized by a nonsystematic rotation (independent of the vibration side), and no significant differences between VBS and control conditions were observed. 


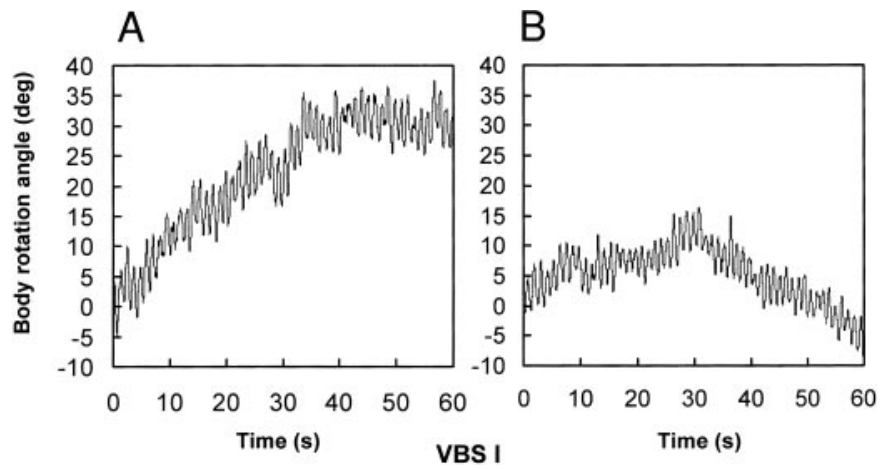

FIG. 3. Two examples from 2 subjects of the effects induced by vibration on the left before stepping-in-place (VBS). A: vibration effect lasted for more than $30 \mathrm{~s}$ after that the vibrator was off; the direction of rotation was opposite to the vibrated side (same as under VDS condition). After this period the subject stopped rotating and stabilized the body orientation on the final rotation angle. $B$ : after about $30 \mathrm{~s}$, the rotation (opposite to the vibrated side as in $A$ ) was replaced by a stronger rotation in the opposite sense.

ment without major medio-lateral displacement or body rotation. In the vibration conditions, there was an obvious rotation, significant with respect to Control under VDS, in all subjects in the direction opposite to the vibrated side. On average, the rotation was about $70^{\circ}$ and $45^{\circ}$ for vibration to the left or right side, respectively. The angular rotation was different from subject to subject, leading to nonhomogeneity of variance between vibration conditions and Control (Mann-Whitney $U$ test: VDS $1, P<0.001$; VDS r, $P<0.001$ ).
Stepping after 60 s-vibration (VBS)

When the neck vibration was applied prior to stepping, obvious but nonsystematic rotation effects were observed from trial to trial and from subject to subject. The body could initially rotate toward one side (most often the same side as during the corresponding VDS condition) and rotate afterwards toward the opposite side. The latency of the changes in rotation was also variable. The final rotation angle could be therefore positive or negative as a function of these effects (Fig. 2). Two typical cases are shown in Fig. 3. In Fig. 3A, the effect persisted for more than $30 \mathrm{~s}$ after that the vibrator was shut off, the rotation sense being the same as during VDS. After this period, the subject stopped rotate and stabilized the body orientation on the final angle. In Fig. 3B, after a first period of rotation as above, the subject rotated in the opposite sense. The overall mean angular rotation of the subjects was not different from the control condition. However, the two means (control and VBS) reflected different ongoing mechanisms because of the nonhomogeneity of the variances (Levene's test; control and VBS 1: $P<0.001$; control and VBS r: $P<0.01$ ).

\section{Latency of the rotation onset during vibration (VDS)}

The onset of body rotation occurred at a variable interval from vibration onset, different from subject to subject and from trial to trial. The distribution frequency of the rotation latencies is shown in Fig. 4A. In general, the rotation began within the first $5 \mathrm{~s}$ from vibration onset. In about $95 \%$ of cases, the

A

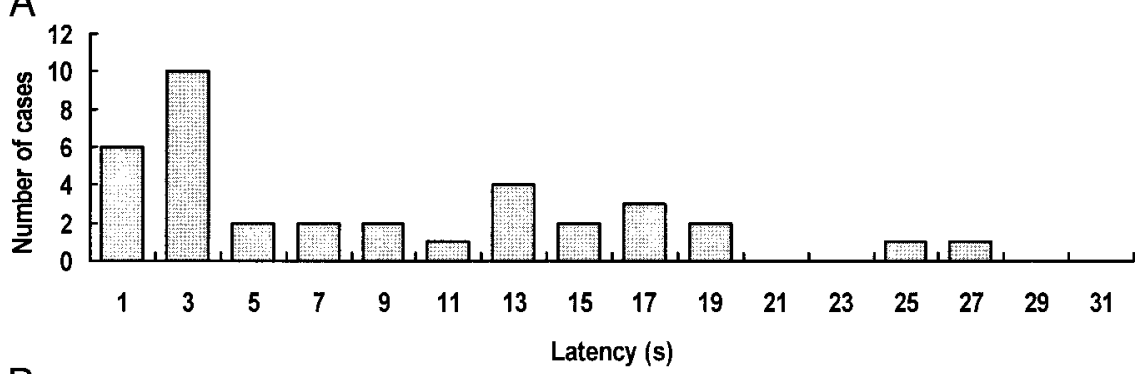

B Latency (s)

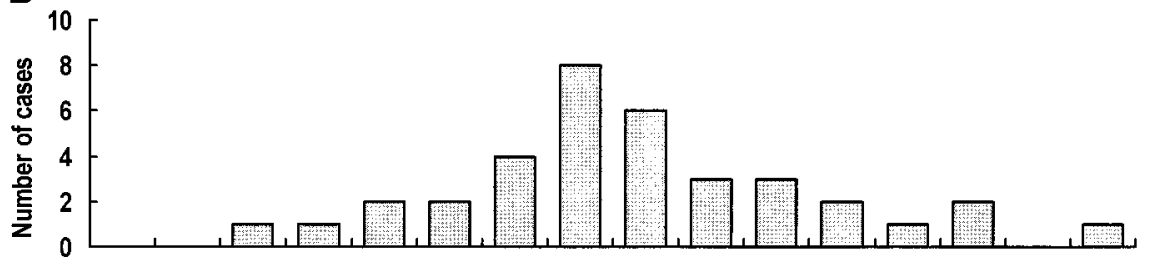

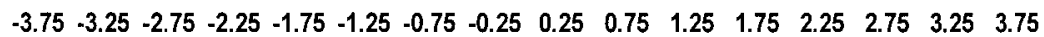

C Velocity (deg/s)

FIG. 4. Latency, velocity, and radius of curvature of body rotation during VDS. A: distribution frequency of the rotation latencies across all trials of all subjects. In general, the rotation began within the first $5 \mathrm{~s}$ from the vibration onset. In the $90 \%$ of cases, the rotations onset had taken place within 20 s. $B$ : distribution frequency of the rotation velocities, in the VDS condition. The mean velocity was $1.08 \%$ s. $C$ : distribution frequency of the lengths of the radius of curvature. distance between the head vertex and the center of the arc of circumference describing the subject's trajectory ranged from 60 to $65 \mathrm{~cm}$ in $70 \%$ of cases.

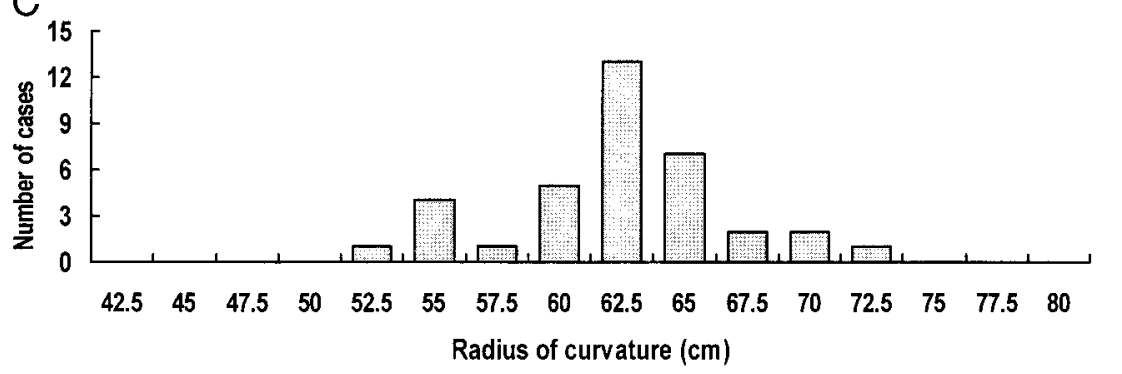


rotation onset had taken place within $20 \mathrm{~s}$ from the start of vibration.

\section{Velocity of the body rotation (VDS)}

Rotation had a variable angular velocity across trials and subjects. However, across all subjects and trials, rotation angle increased steadily as a function of time. Therefore we fitted the time-course of the rotation angle with a straight line. The mean correlation coefficient was close to one regardless of the vibrated side $\left(R^{2}=0.80 \pm 0.05\right)$, indicating strong association between angle variation and time. The slope of the best-fit line was the average rotation velocity. The distribution frequency of the rotation velocities is shown in Fig. $4 B$.

\section{Radius of curvature (VDS)}

The center of the circle fitted to the path traveled by the head vertex and its radius gave an indication of the virtual point, around which the body turned. This was not the same for all subjects, since some rotated around a vertical axis passing through the shoulder, some around an axis placed at some distance from the body. This effect was possibly connected with the concomitant slow forward displacement of the body. In no case, the rotation axis was coincident with the craniocaudal axis. However, Fig. $4 C$ shows that the length of the radius of curvature lie within a remarkably small distance from the body in about $70 \%$ of cases, i.e., from 60 to $65 \mathrm{~cm}$ from the head vertex.

\section{Head-shoulder (yaw) angle (VDS)}

This indicates the orientation of the head with respect to the body; its change might be directly induced by the vibration procedure, accompany the process of rotation, or both. This angle underwent no significant change across conditions (oneway ANOVA: $d f=2,69 ; F=0.038 ; P=0.96$; Fig. $5 A$ ). This was true also for those subjects or trials showing the most ample or rapid body rotations and by collapsing all VDS trials from all subjects no significant association between headshoulder angle and velocity of rotation was found.

\section{Medio-lateral and antero-posterior body inclination (VDS and $V B S$ )}

This would reveal any roll accompanying the rotation during stepping. No consistent changes were seen in the mean body medio-lateral inclination during rotation, regardless of the side vibrated (one-way ANOVA: $d f=2,105 ; F=0.03 ; P=0.97$; Fig. $5 B$, VDS) or the rotation angular velocity or amplitude. The distance between right shoulder and foot was variable in a narrow range across subjects and trials, both under control and vibrated conditions, and after vibration. Some subjects could show sometimes a roll toward the vibrated side, despite their continuous rotation to the opposite side. Due to the variable behavior of body rotation during stepping in VBS, inclinations were inconsistent across trials and subjects and were not systematically calculated. For example, in the trials shown in Fig. 3 ( $A$ and $B$ ), which could be clearly divided into two epochs having almost constant angular velocity, the mean distance varied in a very narrow range. The difference between mean

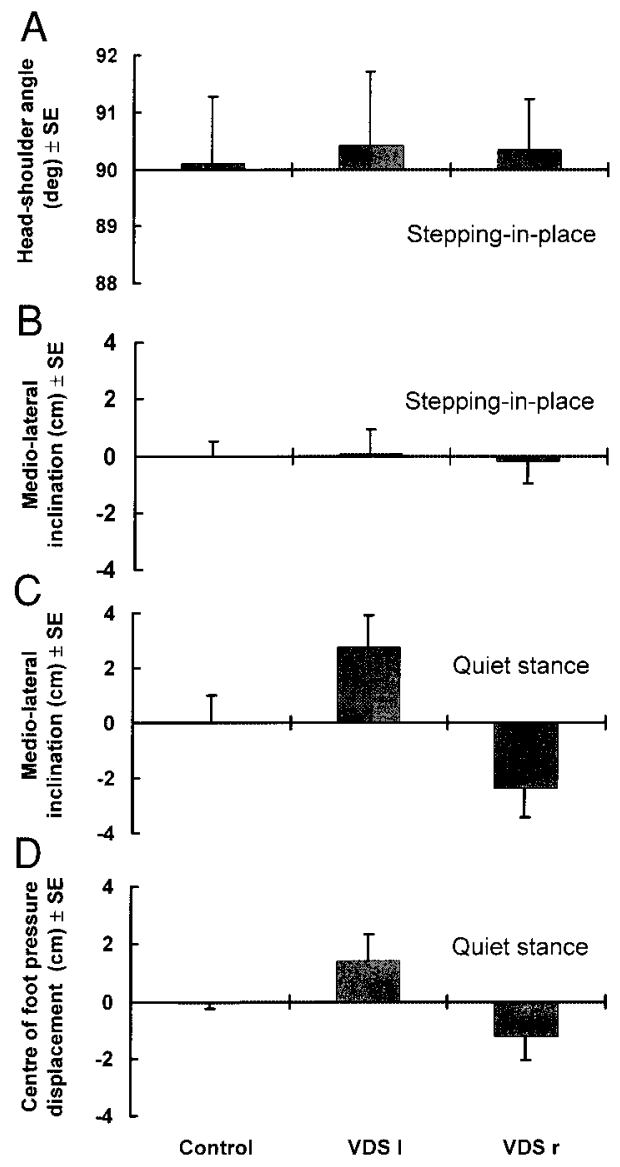

FIG. 5. Lateral neck vibration during stepping $(A$ and $B)$ and stance $(C$ and $D)$. $A$ : head-shoulder angle. During stepping-in-place, no significant changes in the orientation of the head with respect to the body were observed. $B$ : medio-lateral body inclination during stepping. The average horizontal distance between the marker on the right shoulder and on the right malleolus measured under control condition was taken as a reference. During the VDSinduced rotation, no changes in the medio-lateral inclination were observed. $C$ : medio-lateral body inclination during stance. Lack of vibration-induced body inclination during stepping contrasted with the significant and side-dependent effects induced by vibration on stance. $D$ : medio-lateral displacement of the CFP toward the side opposite to vibration. Grand averages ( \pm SE) of all subjects and trials are shown separately for vibrated side (Control; VDS 1: left side vibration during stepping or stance; VDS r: right side vibration during stepping or stance).

distances for trial A was as follows: 1st 40-s epoch versus control, $-0.26 \mathrm{~cm}$; 2nd epoch versus $1 \mathrm{st}$ epoch, $0.2 \mathrm{~cm}$; for trial B: 1st 30-s epoch versus control, $-0.3 \mathrm{~cm} ; 2$ nd versus $1 \mathrm{st}$ epoch, $-0.14 \mathrm{~cm}$. Interestingly, these minor inclinations were analogous to those observed under VDS condition (Fig. 5B), nor was there any correspondence between inclination and rotation sense. The absence of body inclination during stepping contrasted with the effects induced by vibration on stance (Fig. $5 C$ ), where the medio-lateral body inclination indicated a tilt to the side opposite to the vibration, significantly so with respect to control (one-way ANOVA: $d f=2,51 ; F=11.46 ; P<$ 0.05). This was mirrored by the significant CFP displacement on the platform (one-way ANOVA: $d f=2,51 ; F=3.99 ; P<$ 0.05 ; Fig. $5 D$ ), the amplitude of which was in turn compatible with published data (Bove et al. 2001).

No significant antero-posterior shoulder displacements with respect to malleolus were observed across the control and VDS 
conditions (one-way ANOVA: $d f=2,51 ; F=2.54 ; P=0.1$ ). The absence of antero-posterior body inclinations during stepping was confirmed by the results obtained by analyzing the antero-posterior CFP displacements during quiet standing: the antero-posterior displacements of $2.8 \pm 2$ and $1.2 \pm 1.6 \mathrm{~mm}$ for VDS 1 and VDS $r$ conditions, respectively, proved to be nonsignificantly different with respect to the control condition (one-way ANOVA: $d f=2,51 ; F=0.96 ; P=0.39$ ).

\section{Lateral tilt (roll) of head on trunk (VDS)}

This would reveal any lateral roll tilt of head on trunk accompanying the VDS-induced rotation during stepping. Across the subjects, there was no systematic behavior; in some trials the rotation could be accompanied by a minor head roll to one side and in some other trial or subject the reverse could occur. As a matter of fact, neck vibration produced no significant head roll. The mean distance right shoulder-vertex proved to be not different from the mean distance left shoulder-vertex. This was true when these distances were compared between control and VDS trials (one-way ANOVA: $d f=2,51 ; F=$ $2.49 ; P=0.09$ ), and when the rotation period within each VDS trial was compared with the corresponding nonrotation (early) period, across all subjects and trials (paired $t$-test: VDS 1: $t=$ $1.63, P=0.12$; VDS $r: t=-1.23 ; P=0.21)$.

\section{Vibration (VDS) of non-neck muscle}

To ensure that the results were specific to neck activation, we made an experiment in which vibration was applied to a nonneck muscle in three of the six subjects. The muscles (left and right deltoideus medialis and triceps brachii) were not involved in the production of the stepping, but were chosen to induce a strictly lateralized input. The vibration of these muscles produced no significant changes (one-way ANOVA: $d f=$ 5,$48 ; F=0.58 ; P=0.72$ ) in body orientation during stepping (Fig. 6). Figure 6 shows that, at the end of the 1-min period of stepping with vibration of nonneck muscles, the body rotation was of the same order of magnitude of that found under Control conditions (no vibration of any muscle) and certainly negligible with respect to that produced by VDS.

\section{Stepping-in-place, head rotated, no vibration}

When subjects stepped blindfolded with the head rotated to either side, no or nonsystematic body rotations across subjects

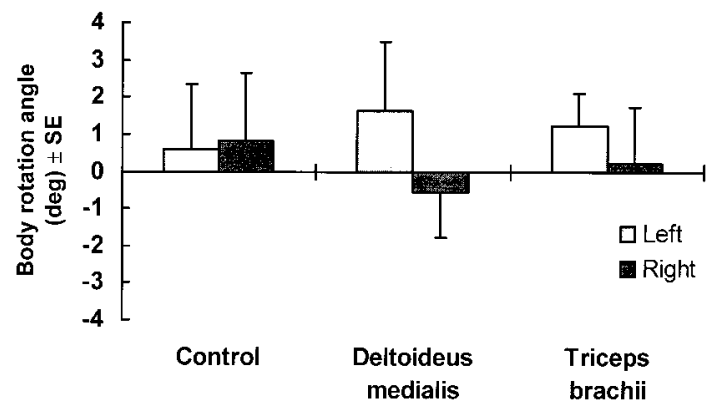

FIG. 6. Effects on the body orientation during stepping-in-place of the vibration of left and right deltoideus medialis and left and right triceps brachii (grand averages \pm SE). Three of the 6 subjects, identified in Fig. 2 by an open circle, open square, and open triangle, were vibrated. No significant changes with respect to the Control condition were observed. and trials were observed. The mean head-shoulder yaw angles were as follows: head in primary position, $0.52 \pm 1.18$; rotation 1: $-49.12 \pm 1.35$; rotation $\mathrm{r}: 51.23 \pm 1.62$. The mean body rotation angles were: head in primary position: $3.49 \mathrm{deg}$ \pm 5.05 ; rotation 1: $10.65 \pm 9.8$; rotation $\mathrm{r}$ : $14.24 \pm 7.06$ (one-way ANOVA: $\mathrm{df}=2,51 ; \mathrm{F}=0.49 ; P=0.62$ ).

\section{I S C U S S I O N}

Lateralized neck muscle vibration induced in all subjects a clear-cut body rotation when applied during stepping-in-place. The rotation was invariably toward the side opposite to the vibrated site, i.e., clockwise on left-sided and counter-clockwise on right-sided vibration. The body rotated around a vertical axis, which intersected the horizontal plane at various distances from the axis passing through the vertex of the head, mostly within arm reach. Of necessity, this implied that rotation was accompanied by a slight component of forward displacement. As a consequence of the interaction of body rotation and displacement, the path traveled by the subjects increased during vibration.

The rotation did not start immediately after switching on the vibrator. The delay varied across subjects from a few seconds to about $10 \mathrm{~s}$ or so. These latencies are much greater than those normally observed in short-latency postural responses to vibration (Andersson and Magnusson 2002; Lekhel et al. 1997), but compatible with the time-course of complex orienting reactions (Grasso et al. 1999; Polonyova and Hlavacka 2001). Thus one could assume that the delayed rotation in response to vibration during stepping-in-place is be the result of a timeconsuming process of integration of neck proprioceptive inflow into the neural circuits' activity responsible for the construction of the spatial references (Karnath et al. 2000). These delays could vary both within and across subjects, indicating a considerable degree of variability in the time necessary to incorporate the asymmetric neck input. Most likely, this variable latency is a cause for the minor and irregular deviation from the straight ahead path observed when short-duration neck muscle vibration was tested on the trajectory of a few-steps linear walk on ground (Bove et al. 2001).

Once initiated, the angular velocity of body rotation was remarkably constant during a given trial, although largely different from subject to subject and trial to trial: the mean angular velocity (VDS) was of the order of $1.08 \%$, so that subjects traveled, on the average, an arch of circumference of about $60^{\circ}$ from rotation onset. The constant velocity speaks against any possible adaptation of the neural structures responsible for the rotation. It would also refute the likelihood that subjects tried to resist the effects of the applied perturbation. This comes as no surprise, since no subject ever noticed the gradual rotation of his or her body and was amazed when, at the end of the session, they were told of the circling behavior.

Since subjects did not normally rotate around their own vertical axis, one would envisage that neck vibration would induce both rotation and forward progression. Progression has been shown to be a consequence of posterior neck vibration (Ivanenko et al. 2000; Lund 1980). Therefore one cannot exclude that the lateral neck vibration used here did activate some spindles of the dorsal cervical muscles. The weight of the two inputs, however, must have been largely in favor of the sternomastoid, since the mean angular velocity of $1.08 \%$, for 
a mean radius of $622 \mathrm{~mm}$, roughly corresponds to a linear velocity of the body along the trajectory of about $\left(1.08^{\circ} / \mathrm{s} \cdot 622\right.$ $\mathrm{mm} \cdot 2 \pi) / 360^{\circ}=11.7 \mathrm{~mm} / \mathrm{s}=0.0117 \mathrm{~m} / \mathrm{s}$, i.e., much less than the $0.24 \mathrm{~m} / \mathrm{s}$ observed on direct dorsal muscle vibration (Ivanenko et al. 2000).

One of our concerns was the possibility that rotation was the mere effect of a lateral tilt induced by the neck vibration, since such stimulation is known to produce a medio-lateral body displacement in standing subjects (Bove et al. 2001; Eklund 1972), and did so in our subjects tested during stance. But lateral tilt observed during quiet stance was not detectable during stepping. Possibly, the enduring stable double-foot base would allow the tilting force (whatever it is) to act up to the point at which it threatens balance. During stepping, when, broadly speaking, one foot at the time is on the ground, equilibrium maintenance becomes the cardinal necessity of the organism and the CNS might call into action neural mechanisms capable of appropriately gating unwanted or perturbing inputs or effects. A related example is represented by the capacity of walking normally despite a continuous, bilaterally applied Achilles tendon vibration (Courtine et al. 2001), an input which severely destabilizes the quiet erect posture. Therefore it is not unlikely that the stepping generator takes care of the necessary accuracy of the intermittent foot placement for maintaining balance and tapers off accordingly the effects of neck vibration. In this sense, the congruity between lateral tilt during stance and side-deviation of walk (Bove et al. 2001 ) is no evidence of a causal relation between the effects of vibration on posture and on gait trajectory. We feel that the "rotation effect" produced by lateral neck vibration during stepping does not operate through the same mechanisms that modify the erect posture. Neither does it require the shift in body center of mass in the mediolateral plane induced by trunk roll, as when voluntary steering is planned early or initiated under time constraints (Patla et al. 1999). Rather, the vibration effect would be related to the capacity of the neck proprioceptive input (in this case an asymmetric lateralized input) to coherently modify the egocentric body-centered coordinate system that allows us to determine our body position with respect to the environment.

A further concern was represented by the possibility that lateral neck muscle vibration would produce head-on-shoulder rotation, thereby indirectly inducing an asymmetrical "natural" input able to favor a curved trajectory of stepping. This is because it is widely held that humans "go where they look," and that head yaw in the sense of the imminent bending of walking trajectory directs the actual turning of the path (Grasso et al. 1996). On the basis of the present data, showing en-block rotation of head and trunk, we would conclude that active head turning in advance of body turning is not obligatory, and that the neural circuits ultimately producing body turning need not produce preliminary head rotation. Admittedly, it can be postulated that when the head actually turns during body steering, a spindle input alike to that elicited by vibration would normally occur. This would help the visual input in guiding locomotor direction and provide a stable frame of reference to control the repositioning of the body in space (Hollands et al. 2001, 2002). Yet, things could be complicated by the fact that the vibration-induced input from the sternomastoid would mimic head rotation toward the vibrated side, since during head turning to one side it is the ipsilateral sternomastoid which is being passively lengthened, while the muscle opposite to the rotation side contracts and shortens (Mazzini and Schieppati 1992). Furthermore, when our subjects stepped in-place with the head deliberately rotated without vibration, no significant effects on body rotation could be detected.

The imbalance of the multiple inputs converging onto the central networks responsible for the "representation" of the sagittal mid-plane would create the conditions for a "new" straight ahead. Imbalance in the vestibular input, produced by irrigating the external auditory meatus with cold water (therefore depressing the activity of the horizontal canal receptor), produces deviation of the walking path toward the side corresponding to the less active labyrinth (Yamamoto et al. 2002). In an analogous way, an abnormal, asymmetric input from the neck would induce deviation of the "straight-ahead" toward the side opposite to the augmented input. Since neck afferents have a modulatory influence on the direction of vestibulo-spinal motor effects in human (Hlavacka and Nijokiktjien 1985) and play a role in shaping the output of the primate vestibular nucleus and its contribution to posture, gaze, and perception (Gdowski and McCrea 2000), the vibration-elicited neck input could modulate the activity of the vestibular nuclei and mimic a rotation of head on shoulder, thereby driving the body to rotate. The neck proprioceptive messages would also contribute, together with visual information of eye position, in determining gaze direction (Han and Lennerstrand 1995). Another asymmetrical "natural" input that is able to favor a curved trajectory of steering can be a systematic change of gaze in the direction opposite to the vibration site (Ivanenko et al. 2000).

One may note that neck muscle vibration has been shown to produce postural, visual, and oculomotor responses also in subjects devoid of vestibular function (at least in the case of dorsal muscles) (Lekhel et al. 1997, 1998; Popov et al. 1999). In passing, this would attenuate the possibility that the effects of the neck vibration reported here would have been produced by mechanical propagation to labyrinthine receptors. Application of a $100-\mathrm{Hz}$ vibratory stimulation to the mastoid bone or neck muscles can indeed evoke nystagmus (Michel et al. 2001), but such effect normally appears in patients with unilateral vestibular dysfunction and hardly so in normal young subjects. Moreover, the vibrator used in that study had an inertial mass of about $1 \mathrm{lb}$, liable to produce nonnegligible parasitic vibration of the skull.

We are not in the position of completely discarding the possibility that the cutaneous stimulation associated with the neck vibration would contribute to the effects observed. Cutaneous fibers presumably activated by vibration have been shown, in the case of foot sole stimulation, to contribute with the leg muscle proprioceptive feedback in controlling human erect posture (Kavounoudias et al. 2001). In the case of the hand, the responses to vibration of the thumb persist when the thumb is anesthetized, suggesting their dependence on the excitation of receptors in the muscle, rather than on cutaneous or joint receptors (Matthews 1984). However, elevation of peripheral inputs by innocuous electrical stimulation of the digital nerves of the digit produces small but significant increases in perceived size of the stimulated part, as assessed by psychophysical methods (Gandevia and Phegan 1999). Stretch of the skin over specific metacarpo-phalengeal joints alters illusions of finger flexion-extension movements induced by vibration over the tendons of the extensor muscles, suggesting 
that the input from cutaneous and muscle spindle receptors is continuously integrated for the perception of finger movements (Collins et al., 2000). We are not aware of similar findings in the case of the neck. Still, electrical galvanic stimulation of neck skin induces modulation of activity in parieto-occipital cortical areas and can interact with vestibular-visual stimulation (Bense et al. 2001). The latter finding would suggest that these effects and interactions may be functionally significant for processing perception and for sensorimotor control. We would conjecture, however, that any vibration-induced cutaneous input from the neck would hardly compete with the spindle input, owing to the much weaker density of skin receptors in the neck compared with foot sole or hand skin, and to the remarkable density of muscle spindles in the neck muscles (see Richmond and Abrahams 1979 in the cat; Boyd-Clark et al. 2002 in man). In this connection, we would here emphasize the genuine role of the input from the neck in this process, since vibration of other muscles had no effect on orientation during stepping-in-place.

The findings obtained with the vibration before stepping-inplace (VBS) contribute an explanation to the erratic effect of vibration preceding straight-ahead locomotion (Bove et al. 2001). The analysis of the time-evolution of the angular changes of the body orientation gave indication of a double behavior. At the beginning of stepping, which occurred on vibration extinction, subjects normally rotated for a while as if their neck were still vibrated. At a variable delay, the sense of rotation changed, as if the effects were now opposite to those observed during actual vibration. long-lasting dynamical modification of posture induced by neck vibration were also observed by Wierzbicka et al. (1998), who described a postvibration shift in posture dependent on vibration side and concluded in favor of a powerful effect of the sustained Ia inflow on the motor system. In our hands, the observed nonsystematic body rotation in the absence of postural effects witnesses a variable weight, from subject to subject, between the persistent though progressively vanishing action of the vibration and the reaction to the abrupt termination of Ia input. The long-lasting postvibration effects on body orientation would further hint at a gradual integration of the neck muscle Ia inflow into the neural circuits' activity responsible for body orientation during locomotion.

All in all, the present results obtained during stepping-inplace, together with the preliminary findings of Bove et al. (2001) obtained during short-distance walk, show that asymmetric neck muscle input plays a major role in body orientation during locomotion, through a mechanisms different from that exerted by vibration on body posture during quiet stance. This confirms and extends the notion that the information related to head rotation on trunk contributes to the definition of a reference system used in the control of human orientation in space.

The authors thank Prof. Paolo Valli for help in the organization of control experiments.

This research was supported by grants from the University of Pavia and Ministero dell' Università e della Ricerca Scientifica e Tecnologica Progetto di Rilevante Interesse Nazionale 1999

\section{REFERENCES}

ANDERSON G AND Magnusson M. Neck vibration causes short-latency electromyographic activation of lower leg muscles in postural reactions of the standing human. Acta Otolaryngol 122: 284-288, 2002.
Bense S, Stephan T, Yousry TA, Brandt T, And Dieterich M. Multisensory cortical signal increases and decreases during vestibular galvanic stimulation (fMRI). J Neurophysiol 85: 886-899, 2001.

Biguer B, Donaldson IM, Hein A, and Jeannerod M. Neck muscle vibration modifies the representation of visual motion and direction in man. Brain 111: 1405-1424, 1988.

Bottini G, Karnath HO, Vallar G, Sterzi R, Frith CD, Frackowiak RS, AND PAUlesu E. Cerebral representations for egocentric space: functionalanatomical evidence from caloric vestibular stimulation and neck vibration. Brain 124: 1182-1196, 2001.

Bove M, Diverio M, Pozzo T, and Schieppati M. Neck muscle vibration disrupts steering of locomotion. J Appl Physiol 91: 581-588, 2001.

Boyadjian A, Marin L, AND Danion F. Veering in human locomotion: the role of the effectors. Neurosci Lett 265: 21-24, 1999.

Boyd-Clark LC, Briggs CA, AND Galea MP. Muscle spindle distribution, morphology, and density in longus colli and multifidus muscles of the cervical spine. Spine 27: 694-701, 2002.

Burke D, Hagbarth KE, Lofstedt L, and Wallin BG. The responses of human muscle spindle endings to vibration of non-contracting muscles. J Physiol (London) 261: 673-693, 1976.

Collins DF, Refshauge KM, And Gandevia SC. Sensory integration in the perception of movements at the human metacarpophalangeal joint. J Physiol (Lond) 529: 2: 505-15, 2000.

Cordo P, Gurfinkel VS, Bevan L, and Kerr G.K. Proprioceptive consequences of tendon vibration during movement. J Neurophysiol 74: 1675$1688,1995$.

Courtine G, Pozzo T, Lucas B, and Schieppati M. Continuous leg muscle vibration is not detrimental to human walk. Brain Res Bull 55: 107-115, 2001

CRASKE B. Perception of impossible limb positions induced by tendon vibration. Science 196: 71-73, 1977.

EKLUND G. General features of vibration-induced effects on balance. Uppsala J Med Sci 77: 112-124, 1972.

FukUDA T. The stepping test. Two phases of the labyrinthine reflex. Acta Otolaryngol 50: 26, 1959.

Gandevia SC And Phegan CM. Perceptual distortions of the human body image produced by local anaesthesia, pain and cutaneous stimulation. J Physiol (Lond) 514: 609-616, 1999.

GDOWSKI GT AND MCCREA RA. Integration of vestibular and head movement signals in the vestibular nuclei during whole body rotation. J Neurophysiol 82: 436-449, 1999.

GDOwSKI GT AND MCCREA RA. Neck proprioceptive inputs to primate vestibular nucleus neurons. Exp Brain Res 135: 511-526, 2000.

Goodwin GM, McCloskey DI, and MatThews PB. The contribution of muscle afferents to kinaesthesia shown by vibration induced illusions of movement and by the effects of paralysing joint afferents. Brain 95: 705748, 1972.

Grasso R, Glasauer S, Takei Y, and Berthoz A. The predictive brain: anticipatory control of head direction for the steering of locomotion. Neuroreport 7: 1170-1174, 1996.

GRASSO R, IVANENKO Y, AND LACQUANITI F. Time course of gaze influences on postural responses to neck proprioceptive and galvanic stimulation in humans. Neurosci Lett 273: 121-124, 1999.

GuRFInKEl VS, IVANENKo YP, LEVIK YS and BABAKova IA. Kinaesthetic reference for human orthograde posture. Neuroscience 68: 229-243, 1995.

HAN Y AND LENNERSTRAND G. Eye movements in normal subjects induced by vibratory activation of neck muscle proprioceptors. Acta Ophthalmol Scand 73: 414-416, 1995.

Hlavacka F AND NijokiktJien C. Postural responses evoked by sinusoidal galvanic stimulation of the labyrinth. Influence of head position. Acta Otolaryngol 99: 107-112, 1985.

Hollands MA, PATLA AE, AND VickeRs N. Look where you're going!: gaze behaviour associated with maintaining and changing the direction of locomotion. Exp Brain Res 143: 221-230, 2002.

Hollands MA, SoRENSEn KL, AND PATla AE. Effects of head immobilization on the coordination and control of head and body reorientation and translation during steering. Exp Brain Res 140: 223-233, 2001.

IVANENKO YP, GRASSO R, AND LACQUANITI F. Effect of gaze on postural responses to neck proprioceptive and vestibular stimulation in humans. J Physiol (Lond) 519: 301-314, 1999.

IVANENKO YP, GRASSO R, AND LACQUANITI F. Neck muscle vibration makes walking humans accelerate in the direction of gaze. J Physiol (Lond) 525: 803-814, 2000. 
KARNATH HO. Subjective body orientation in neglect and the interactive contribution of neck muscle proprioception and vestibular stimulation. Brain 117: 1001-1012, 1994.

Karnath HO, Christ K, AND HartJe W. Decrease of controlateral neglect by neck muscle vibration and spatial orientation of trunk midline. Brain 116: 383-396, 1993.

KARnATH HO, KoncZAK J, AND DichGans J. Effect of prolonged neck muscle vibration on lateral head tilt in severe spasmodic torticollis. J Neurol Neurosurg Psychiatry 69: 658-660, 2000.

KARNATH HO, Sievering D, AND FETTER M. The interactive contribution of neck muscle proprioception and vestibular stimulation to subjective 'straight ahead' orientation in man. Exp Brain Res 101: 140-151, 1994.

Kavounoudias A, Gilhodes JC, Roll R, and Roll JP. From balance regulation to body orientation: two goals for muscle proprioceptive information processing? Exp Brain Res 124: 80-88, 1999.

Kavounoudias A, Roll R, AND Roll JP. Foot sole and ankle muscle inputs contribute jointly to human erect posture regulation. J Physiol (Lond) 532: 869-878, 2001.

Lekhel H, Popov K, Anastasopoulos D, Bronstein A, Bhatia K, Marsden CD, AND GREsty M. Postural responses to vibration of neck muscles in patients with idiopathic torticollis. Brain 120: 583-591, 1997.

Lekhel H, Popov K, Bronstein A, And Gresty M. Postural responses to vibration of neck muscles in patients with uni- and bilateral vestibular loss. Gait Posture 7: 228-236, 1998.

LuND S. Postural effects of neck muscle vibration in man. Experientia 36 : 1398, 1980.

LuND S AND BRoBERG C. Effects of different head positions on postural sway in man induced by a reproducible vestibular error signal. Acta Physiol Scand 117: 307-309, 1983.

MatTHEws PB. Evidence from the use of vibration that the human long-latency stretch reflex depends upon spindle secondary afferents. J Physiol (Lond) 348: 383-415, 1984.

MAZZINI L AND SchiEPPATI M. Preferential activation of the sternocleidomastoid muscles by the ipsilateral motor cortex during voluntary rapid head rotations in humans. In: The Head-Neck Sensory Motor System, edited by Berthoz A, Vidal PP, and Graf W. Oxford: Oxford University Press, 1992, p. $597-600$

Michel J, Dumas G, Lavielle J-P, and Charachon R. Diagnostic value of vibration-induced nystagmus obtained by combined vibratory stimulation applied to the neck muscles and skull of 300 vertiginous patients. Rev Laryngol Otol Rhinol 122: 89-94, 2001.

Naito E, Ehrsson HH, Geyer S, Zilles K, And Roland PE. Illusory arm movements activate cortical motor areas: a positron tomography study. J Neurosci 19: 6134-6144, 1999.

Patla AE, Adkin A, AND Ballard T. Online steering: coordination and control of body center of mass, head and body reorientation. Exp Brain Res 129: 629-634, 1999.
Polonyova A AND Hlavacka F. Human postural responses to different frequency vibrations of lower leg muscles. Physiol Res 50: 405-410, 2001.

Popov KE, LeKhel H, FAldon M, Bronstein AM, And Gresty MA. Visual and oculomotor responses induced by neck vibration in normal subjects and labyrinthine-defective patients. Exp Brain Res 128: 343-352, 1999.

Richmond FJ AND ABRAHAMS VC. What are the proprioceptors of the neck? Prog Brain Res 50: 245-254, 1979.

Roll JP AND Roll R. From eye to foot. A proprioceptive chain involved in postural control. In: Posture and Gait, edited by Amblard B, Berthoz A, and Clarac F. Amsterdam: Elsevier, 1988, p. 155-164.

RoLl JP AND VEDEL JP. Kinaesthetic role of muscle afferents in man, studied by tendon vibration and microneurography. Exp Brain Res 47: 177-190, 1982.

Roll JP, VEDEL JP, AND RIBOT E. Alteration of proprioceptive messages induced by tendon vibration in man: a microneurographic study. Exp Brain Res 76: 213-222, 1989a.

Roll JP, Vedel JP, AND Roll R. Eye, head and skeletal muscle spindle feedback in the elaboration of body references. Prog Brain Res 80: 113-123, $1989 b$.

RUDOMIN P. Selectivity of presynaptic inhibition: a mechanism for independent control of information flow through individual collaterals of single muscle spindle afferents. Prog Brain Res 123: 109-117, 1999.

SCHIEPPATI M. The Hoffmann reflex: a means of assessing spinal reflex excitability and its descending control in man. Prog Neurobiol 28: 345-376, 1987.

SCHIEPPATI M AND CRENNA P. From activity to rest: gating of excitatory autogenetic afferences from the relaxing muscle in man. Exp Brain Res 56: 448-457, 1984

Smetanin BN, Popov KY, And Shlykov VY. Postural responses to vibrostimulation of the neck muscle proprioceptors in man. J Neurophysiol 25: 101-108, 1993.

Strupp M, Arbusow V, Borges Pereira C, Dieterich M, and Brandt T. Subjective straight-ahead during neck muscle vibration: effects of ageing. Neuroreport 10: 3191-3194, 1999.

Tarantola J, Nardone A, TAcchini E, And Schieppati M. Human stance stability improves with the repetition of the task: effect of feet position and visual condition. Neurosci Lett 228: 75-78, 1997.

TAYLOR J AND MCCLOSKEY DI. Illusions of head and visual target displacement induced by vibration of neck muscles. Brain 114: 755-759, 1991.

Ushio N, Hinoki M, Baron JB, Gagey PM, And Meyer J. The stepping test with neck torsion: proposal of a new equilibrium test for cervical vertigo. Prat Otol (Kyoto) 69: 1369-1379, 1976.

Yamamoto K, Mamoto Y, Imai T, Hirasaki E, and Kubo T. Effects of caloric vestibular stimulation on head and trunk movements during walking. Gait Posture 15: 274-281, 2002.

WiERZBickA MM, GILHODES JC, AND RolL JP. Vibration-induced postural posteffects. J Neurophysiol 79: 143-150, 1998. 